\title{
Prism adaptation in left-handers
}

\author{
Gordon M. Redding • Benjamin Wallace
}

Published online: 17 May 2011

(C) Psychonomic Society, Inc. 2011

\begin{abstract}
Two experiments with left-handers examined the features of prism adaptation established by previous research with right-handers. Regardless of handedness, (1) rapid adaptation occurs in exposure pointing with developing error in the opposite direction after target achievement, especially with early visual feedback in target pointing; (2) proprioceptive or visual aftereffects are larger, depending on whether visual feedback is available early or late, respectively, in target pointing; (3) the sum of these aftereffects is equal to the total aftereffect for the eye-hand coordination loop; (4) intermanual transfer of visual aftereffects occurs only for the dominant hand; and (5) visual aftereffects are larger in left space when the dominant hand is exposed to leftward displacement. A notable handedness difference is that, while transfer of proprioceptive aftereffects only occurs to the nondominant hand in righthanders, transfer occurs in both directions for left-handers, but regardless of handedness, such transfer only occurs when the exposed hand is tested first after exposure. A discussion then focuses on the implications of these data for a theory of handedness.
\end{abstract}

Keywords Prism adaptation · Handedness · Intermanual transfer $\cdot$ Motor control · Spatial mapping $\cdot$ Spatial alignment

Intermanual transfer of prism adaptation is asymmetric in right-handed subjects: That is, transfer of proprioceptive

G. M. Redding $(\bowtie)$

Department of Psychology, Illinois State University, Campus Box 4620,

Normal, Illinois 61790-4620, USA

e-mail: gredding@ilstu.edu

B. Wallace

Cleveland State University,

Cleveland, Ohio 44115, USA adaptation is larger from the dominant right hand to the nondominant left hand, while transfer of visual adaptation is larger from the nondominant left hand to the dominant right hand (Redding \& Wallace, 1988b). The purpose of the present experiments was to test hypotheses about how intermanual transfer may be related to handedness by examining prism adaptation in lefthanded subjects.

While prism adaptation in right-handed subjects has been systematically investigated for well over 50 years (Held \& Hein, 1958; Redding \& Wallace, 1997a, 2006; von Helmholtz, 1909/1962; Welch, 1978, 1986), nothing is known about prism adaptation in left-handed subjects. To our knowledge, there have been no investigations with lefthanders. It was necessary, therefore, to determine whether prism adaptation in left-handers is similar to that in righthanders. Such similarity would be a precondition for testing whether asymmetric intermanual transfer is related to handedness.

Below, we first identify the general characteristics of prism adaptation for right-handers, to provide a basis for comparison with left-handers. Then, in two experiments, we develop the handedness hypotheses about intermanual transfer that we tested with left-handed subjects. Experiment 1 examined transfer of proprioceptive adaptation, and Experiment 2 examined transfer of visual adaptation.

\section{Prism adaptation in right-handers}

In the first, and still typical, prism adaptation procedure (Redding \& Wallace, 2006; von Helmholtz, 1909/1962), a person wears a goggle or spectacle frame holding wedge prisms that displace the visual field, for example, in the rightward direction, and then points toward visual targets with the right hand. Initially, pointing errors are made to the 
right of the target. However, such errors diminish over repeated trials with visual feedback, and disappear in as few as 15 trials. The person has "adapted" to the prismatic displacement.

A significant feature of this exposure adaptation, the reduction of the direct effect of the prisms, is that if targetpointing exposure is continued beyond target achievement, pointing errors opposite to the initial direction appear (Redding \& Wallace, 1993, 1997b), especially when visual feedback is given early in the pointing movement (Redding \& Wallace, 1993). This overcompensation is one of several sources of converging evidence indicating that while ordinary error correction is responsible for rapid adaptation, exposure adaptation also involves another, slower adaptive process that produces antiadaptive behavior when increasingly nonadaptive error correction strategies continue to be applied.

The fact that prism exposure evokes at least two very different and dissociable kinds of adaptive processes, ordinary error correction and extraordinary spatial realignment, has been repeatedly demonstrated for prismatic displacement (Bedford, 1989, 1993a, 1993b, 1999; Redding \& Wallace, 1993, 1996, 2001, 2002, 2006; see also Newport \& Jackson, 2006; Redding, Clark, \& Wallace, 1985; Redding, Rossetti, \& Wallace, 2005; Redding \& Wallace, 1997a, 2006; Weiner, Hallett, \& Funkenstein, 1983) and has recently been shown for virtual displacement (Simani, McGuire, \& Sabes, 2007). The spatial misalignment between the origins of the visual eye-head and proprioceptive handhead spatial maps, for example, is normally removed by transformational constants applied to control signals between sensory-motor systems. In this way, spatial maps are aligned, and signals between different maps are expressed in a common coordinate system. Prismatic displacement disturbs this spatial alignment and enables study of the realignment process.

Assessment of this second adaptive process requires that the experimental procedure be augmented by measures of adaptation obtained after prism exposure when error correction is discontinued: that is, the aftereffects of adaptation. Three kinds of aftereffect measures are commonly obtained: (1) total adaptation, or the total shift in straight-ahead for the eye-hand coordination loop exercised during exposure, is measured by visual target pointing without displacement and without feedback; (2) proprioceptive adaptation, or the proprioceptive shift in straightahead for the hand-head component of the eye-hand coordination loop, is measured by pointing straight ahead without vision and without feedback; and (3) visual adaptation, or the visual shift in straight-ahead for the eye-head component of the eye-hand coordination loop, is measured by nonmanual (e.g., verbal) adjustment of a visual target to straight-ahead without involvement of the exposed limb and without feedback. Aftereffect measures of the shift in straight-ahead are obtained by a comparison of postexposure performance with preexposure baselines.

Realignment aftereffects occur in either or both of the proprioceptive hand-head sensory-motor system and the visual eye-head sensory-motor system (Cohen, 1967; Craske, 1967; Harris, 1963, 1965; Hay \& Pick, 1966; Redding \& Wallace, 1988a, 1988b; Rock \& Harris, 1967). Proprioceptive aftereffects are predominant when sight of the pointing hand during exposure occurs early in the pointing movement, while visual aftereffects predominate when such visual feedback is delayed until near the terminus of the pointing movement (Canon, 1970; Cohen, 1967; Kelso, Cook, Olson, \& Epstein, 1975; Redding \& Wallace, 1990, 1992, 1994, 2000; Uhlarik, 1973; Uhlarik \& Canon, 1971). Proprioceptive aftereffects are also larger when the rate of pointing during exposure is high, while visual aftereffects are larger with low-exposure pointing rates (Choe \& Welch, 1974; Redding \& Wallace, 1990, 1992, 1994).

The algebraic sum of the visual and proprioceptive aftereffects usually equals the total aftereffect measured in the complete eye-hand coordination loop exercised during exposure (Hay \& Pick, 1966; McLaughlin \& Webster, 1967; Templeton, Howard, \& Wilkinson, 1974; Wallace, 1977; Wallace \& Redding, 1979; Wilkinson, 1971). Exceptions to such additivity can arise from associative transfer of ordinary error-corrective processes deployed during exposure to similar aftereffect assessment tasks (Beckett, 1980; Redding \& Wallace, 1978, 2006; Wallace, 1977; Welch, 1971; Welch, Choe, \& Heinrich, 1974: see also Wallace, 1974; Welch \& Rhodes, 1969).

The first experiment was designed to test for overcompensation when exposure is extended beyond target achievement and for the dependence of proprioceptive aftereffects upon early visual feedback. The second experiment was designed to test for visual aftereffects with delayed visual feedback. Both experiments assessed the additivity of the component aftereffects to measure whether they equaled the total aftereffect. In addition, Experiment 1 investigated intermanual transfer of proprioceptive aftereffects, and Experiment 2 studied intermanual transfer of visual aftereffects. Specific transfer hypotheses are developed with the report of each experiment.

\section{General method}

The experiments reported here were the left-hander analogues of previous experiments with right-handers. The method employed in Experiment 1 was similar to that used by Redding and Wallace (2008) to study proprioceptive transfer, except that left-handed subjects were tested. 
Experiment 2 employed the same method used by Redding and Wallace (2009) to study visual transfer, except for the change in the handedness of the subjects. Experiment 1 employed early visual feedback to produce predominantly proprioceptive adaptation, while Experiment 2 delayed sight of the hand until the terminus of the pointing movement to produce primarily visual adaptation. In both experiments, the total aftereffect for the exposed eye-hand coordination loop was measured in order to permit examination of the additivity of the proprioceptive and visual components. Also in both experiments, unexposed as well as exposed hands were tested in order to enable measurement of aftereffect transfer. Terminal error in exposure pointing was also measured in order to permit assessment of the direct effects for left-handers.

\section{Subjects}

All subjects were left-handed student volunteers at Cleveland State University. A version of the Edinburgh Handedness Inventory (Oldfield, 1971) was used to assess handedness. All subjects were asked with which hand they throw a ball, hold a knife, write, and push a broom. If they responded "left" to all four of these questions, they were deemed left-handers. Subjects had self-reported normal vision or vision that was corrected to normal by contact lenses. No other subject demographics were recorded. These subjects were treated in accordance with the "Ethical Principles of Psychologists and Code of Conduct" (American Psychological Association, 1992).

\section{Apparatus}

The apparatus is illustrated in Fig. 1. This structure consisted of a two-layer, rectangular, wooden, box-like frame ( $24 \mathrm{~cm}$ high, $105 \mathrm{~cm}$ wide, and $74 \mathrm{~cm}$ deep) placed on a table and open on the side facing the subject. All visible surfaces were homogeneously white, thereby allowing only minimally visible landmarks, and nothing beyond the apparatus was visible to the subjects. Attached to the front of the apparatus was a Marietta Apparatus Co. (Marietta, OH) Model No. 75-A-2-12 chinrest. Subjects sat at the apparatus, with head in the chinrest, and made sagittal pointing movements toward a target located on the back vertical surface of the apparatus.

When subjects placed their arms within the structure, on the lower layer, the arm was not visible. In this area, during baseline and aftereffect tests before and after prism exposure, respectively, limb position was measured by determining the limb's position along a calibrated 180-deg arc. The origin of the measurement scale was below the subject's chin, near the trunk. Thus, limb position was measured as the angle made by the hand relative to the

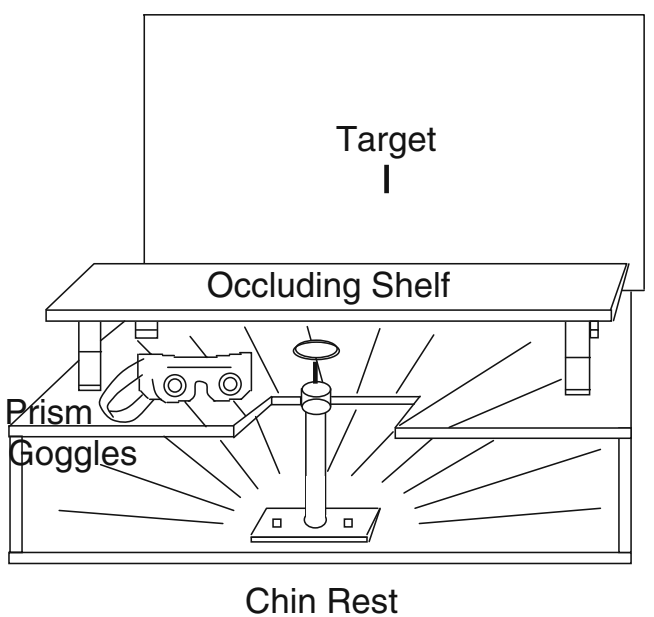

Fig. 1 The apparatus. Subjects were seated, with head constrained by a chinrest and wearing goggles that held the prisms. For realignment pretests and posttests, the subject's right arm was not visible on the lower level of the apparatus, a target line was introduced on the back vertical surface, and the starting position for sagittal pointing was the base of the chinrest. For exposure, subjects placed their arm on the top level and, after leaving the starting position against the chinrest support, the distal half of the movement path was visible (Exp. 1), or with the occluding shelf in place, sight of the limb was occluded over the entire movement path until the tip of the finger was seen beyond the occluding board (Exp. 2). Measurement scales, not visible to the subject, originating at the chinrest vertical on both levels were used to record performance

body midline. The starting position for sagittal pointing during alignment tests before, during, and after prism exposure was the base of the chinrest.

During exposure, subjects placed their arm upon the upper layer. A second measurement scale for recording performance was positioned on the surface of this upper layer and was not visible to the subject. The starting position for sagittal pointing during prism exposure was the vertical support of the chinrest. For Experiment 1, the subject's limb was visible for the distal half of the pointing movement toward the target, while the proximal half of the movement path was occluded by the prism-bearing goggles: That is, visual feedback was available early over the distal half of the movement path. For Experiment 2, an occluding shelf was placed at about nose level and adjusted so that only the subject's fingertip was visible at the completion of the pointing movement toward the target: That is, visual feedback was available only late, at the terminus of the pointing movement. Assuming an average arm length of $50 \mathrm{~cm}$, in Experiments 1 and 2 the limb was visible for the distal $24 \mathrm{~cm}$ and $2 \mathrm{~cm}$ of the pointing movement, respectively. Note that the target was, on average, about $24 \mathrm{~cm}$ beyond the subjects' reach.

Subjects wore welder's safety goggles with a Risley prism mounted in each eyepiece that could be set to produce lateral (rightward or leftward) prismatic displace- 
ment of the optic array varying from 0 to 30 diopters (1 diopter $=0.57 \mathrm{deg}$ arc) and that afforded binocular vision. Each circular eyepiece subtended a visual angle of approximately $30 \mathrm{deg}$, and the width of the binocular field was approximately $45 \mathrm{deg}$.

\section{Design}

A mixed design was employed for both experiments: Order of Aftereffect Tests and Exposed Hand were the betweensubjects factors, while Exposure Trials and Aftereffect Tests were the within-subjects factors. Subjects were randomly assigned to groups, with the exception that in Experiment 2 the right-hand exposure groups were tested subsequently to left-hand exposure groups. Measures of terminal limb position in sagittal target pointing were recorded for each exposure trial.

Before and after exposure to one of the between-subjects conditions, subjects performed five tests of perceived straight-ahead, without prismatic displacement and without visual feedback or knowledge of the results. These tests were designed to provide for baseline and aftereffect measures of change in visual straight-ahead (visual shift), straight-ahead limb position (proprioceptive shift) for both the exposed and unexposed limbs, and straight-ahead for eye-hand coordination with both the exposed and unexposed limbs (total shift). Different orders of these tests were used in the two experiments.

\section{Procedure}

Upon arrival in the laboratory, each subject received a brief description of their participation, including the fact that perceptual-motor coordination tests would be given before and after a period of eye-hand coordination activity while looking through the prisms. Subjects were not informed of the nature of the distortion, only that the prisms might affect their performance and that they should try to perform the task as accurately as possible. The subject was seated in a chair before the apparatus with head positioned in the chinrest and wearing the prism-bearing goggles. The subject was then asked to perform each of the preexposure aftereffect baseline tests.

The visual shift test involved no limb movement. Instead, the experimenter introduced a moving, visible, vertical target line $(0.2 \times 8 \mathrm{~cm})$ on the back vertical surface of the upper layer of the apparatus and at the subject's eye level. When the experimenter moved this target laterally across the subject's visual field at a rate of approximately $3 \mathrm{~cm} / \mathrm{s}$, the subject verbally indicated when the target appeared to be straight ahead of the nose. The stopping point, indicating straight ahead, was read from a scale not visible to the subject (see Fig. 1 and the associated text).
The subject's eyes were closed when the line was moved to a starting position. The duration of a test trial varied between approximately 5 and $10 \mathrm{~s}$, depending on the distance from the random starting position to the position that a subject judged to be straight ahead of the nose. The prisms were set to zero diopters for this test, as was true for all pre-post exposure test situations. Because this test was referenced to the head, the difference between this baseline preexposure measure and performance on the same test administered after prism exposure could be assumed to provide an aftereffect measure of the change in straightahead for the visual (eye-head) system. ${ }^{1}$

In Experiment 1, 10 test trials were given, half starting randomly in the left and right visual fields. In Experiment 2, 10 trials started with the target randomly placed in the right half of the visual field, and 10 trials started randomly in the left visual field. The order of the left- and rightstarting-position trial blocks was counterbalanced.

The proprioceptive shift test required the subjects to place their to-be-exposed hand on the lower layer of the test apparatus, against the base of the chinrest, and to point sagittally to the position in space believed to be straight ahead of their nose. Responses were read from a scale not visible to the subject (see Fig. 1 and the associated text). This task was performed with vision occluded by a blindfold placed over the prism-bearing goggles. The test was performed 10 times, each test requiring $3 \mathrm{~s}$. The test for transfer of proprioceptive shift to the unexposed limb was identical to the previous test, except that the unexposed limb was tested, providing a measure of transfer to the unexposed hand-head proprioceptive system. Because these tests were referenced to the head, a change in performance after prism exposure could be assumed to provide an aftereffect measure of the changes in straightahead for the exposed hand-head proprioceptive system and the unexposed hand-head proprioceptive system. ${ }^{2}$

\footnotetext{
${ }^{1}$ The term "visual shift" is used to designate adaptive change in the eye-head system that has phenomenal consequences for visual perception. Such a change may affect either the retinal local sign or the direction of gaze (see, e.g., Crawshaw \& Craske, 1974; Harris, 1980). The current development of the theory does not permit a comparison between these two possible accounts of visual change (but see Redding \& Wallace, 1997a), and the aftereffect test for visual shift used in the present experiment was sensitive to either or both kinds of change.

2 The term "proprioceptive" is used to designate any adaptive change in position sense at the joints between the head and the hand, or even the fingers. We assume that the hand-head system is hierarchically organized (Redding \& Wallace, 1992, 1993, 1997a, 2002; see also Jeannerod, 1988) such that any proximal change extends to positioning of more distal joints. The present proprioceptive shift test was designed to detect all position sense changes in the hand-head system. Indeed, we think of the spatial map for the limb as being defined by the combination of joint positions (e.g., Churchland, 1986; Redding \& Wallace, 2002).
} 
The total shift test was similar to the proprioceptive shift test, except that the subject was not blindfolded and pointed with the to-be-exposed hand toward the target line that had been used for the visual shift test, but now the line was stationary and physically straight ahead. Responses were read from a scale not visible to the subject (see Fig. 1 and the associated text). During this test, the subject viewed the target with no prismatic displacement, and the accuracy of pointing was not known to the subject, because the hand was on the lower level of the apparatus and not visible. Ten measures were taken, each requiring $3 \mathrm{~s}$. The transfer of total shift test was identical, except that the unexposed limb was tested. Because these tests involved the complete eyehand coordination loop, the difference between this baseline preexposure measure and performance on the same test administered after prism exposure could be assumed to provide an aftereffect measure of the change in straight-ahead for either or both of the visual (eye-head) and proprioceptive (exposed hand-head and unexposed hand-head) systems.

Following establishment of the realignment baselines, the prisms were set to displace the visual field 20 diopters (11.4 deg), and subjects were randomly assigned to one of the exposure conditions, defined by a factorial combination of the between-subjects variables. Note that the subject's limb was not visible in the exposure starting position against the chinrest vertical support (see Fig. 1). This was a necessary condition for adaptation aftereffects (Redding \& Wallace, 1996, 1997b).

The displaced visual field contained the stationary and straight-ahead target line used in the pretests, but now optically displaced $11.4 \mathrm{deg}$. Subjects attempted sagittal pointing movements toward the visible, vertical target line, and they received either early visual feedback during the distal half of the pointing movement (Exp. 1) or delayed visual feedback by sight of the fingertip at the end of the pointing movement (Exp. 2). Upon completion of an outward pointing movement, subjects immediately reversed direction, returned to the starting position, then pointed again toward the target, and so on, with continuous out-and-back movements. Each exposure trial required $3 \mathrm{~s}$.

The terminal accuracy in pointing at the target was visually observed on a measurement scale not visible to the subject (see Fig. 1 and the associated text) and was recorded by the experimenter for each subject on each trial. These observations were made when the subject's finger paused briefly, signaling outward movement completion. Movements after a pause but not in the opposite (return) direction were considered secondary corrections. Such secondary movements were discouraged and, in fact, appeared only infrequently. Of course, visual information about the terminal error on previous trials (i.e., knowledge of the results) could have been used to initiate the next outward movement toward the target on the next trial.

After the exposure period, the prisms were reset to zero diopters; the subjects were told that any distortion was no longer present, and the aftereffect tests were repeated. The predicted posttest adaptive change from the pretest baseline was opposite the direction of prismatic displacement for the tests requiring limb movement, but in the direction of prismatic displacement for the visual shift test (Redding et al., 2005; Redding \& Wallace, 1988a, 1998, 2000; Welch, 1978).

All measurements were to the nearest degree. Throughout the experiment, each subject's head was constrained by the chinrest (see Fig. 1), and the head was visually monitored by the experimenter to correct any changes in position.

Pointing movements, during both exposure and tests, were paced by a metronome set to beat every $1.5 \mathrm{~s}(0.67$ beats/s); that is, one trial every $3 \mathrm{~s}$. This method controlled movement speed and assured movements slow enough for an accurate measurement of terminal pointing error. Such slow, sound-paced movement was also a necessary condition for visual adaptation (Redding \& Wallace, 1992, 1993, 1994, 2000). Movements paced by an external auditory signal generally show "negative asynchrony," in which movement completion occurs slightly before the pacing signal (Aschersleben, Drewing, \& Stenneken, 2002), followed by a pause while waiting for the signal, before beginning the return movement.

Measurement in previous studies established that subjects typically paused between 0.025 and $0.100 \mathrm{~s}$ at the end of each movement segment, producing an average movement speed of between 34 and $36 \mathrm{~cm} / \mathrm{s}$, with the tip of the pointing finger visible (outward and backward, including the pause toward the target) for about $1.5 \mathrm{~s}$ for early feedback (Exp. 1) and between 0.1 and $0.2 \mathrm{~s}$ for late feedback (Exp. 2).

\section{Experiment 1: Proprioceptive transfer}

Intermanual transfer of proprioceptive aftereffects is a particularly interesting point of comparison for adaptation in right- and left-handed subjects, because transfer seems to be related to hand dominance. For right-handers, little or no intermanual transfer appears when the proprioceptive shift test is used for assessment, especially with slow rates of exposure pointing (Choe \& Welch, 1974; Harris, 1963; Templeton et al., 1974; but see Wallace, 1978), and any partial transfer is asymmetric-larger from the dominant right hand to the nondominant left hand (Redding \& Wallace, 1988b).

Importantly, recent research has shown that proprioceptive transfer depends on the order in which the exposed and 
unexposed hands are tested after exposure (Redding \& Wallace, 2008; see also Cohen, 1973; Wallace, 1978); transfer only occurs from the dominant right hand to the nondominant left hand, and only when the dominant right hand is tested first after exposure. When the dominant right hand is exposed, the transfer is positive, but when the nondominant left hand is exposed, the transfer is negative. In the first case, transfer from the exposed right hand produces an aftereffect in the unexposed left hand, but in the second case, transfer from the unexposed right hand reduces the aftereffect in the exposed left hand.

These results for right-handers suggest that motor control of one hand is independent of control of the other, at least for purposes of spatial alignment. Indeed, Prablanc, Tzavaras, and Jeannerod (1975) dramatically demonstrated this independence by showing that each hand can adapt simultaneously to opposite directions of displacement! However, the right-hand spatial map is also connected with the left-hand spatial map.

This connection from the right to the left hand normally serves to establish the left-hand position within the righthand task-work space. A task-work space is a portion of the entire egocentric space within which the current task is performed, selected to minimize processing load and maximize task performance (Redding \& Wallace, 2001). For right-handers, the task-work space is set by the dominant right hand, and the nondominant left hand is registered within this right-hand task-work space. That is, a control signal is sent specifying the position of the left hand within the right-hand task-work space. Such calibration of the left hand only occurs when the right hand is actively engaged in a specific task, and only after the work space for the right hand has been established.

However, as illustrated in Fig. 2, when the spatial maps for the two hands are misaligned, the calibration command produces spatial discordance and realignment of the left-

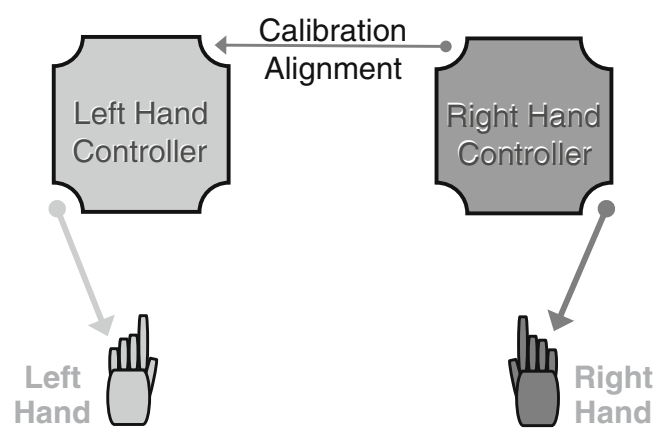

Fig. 2 Intermanual processing connection for right-handers. The directional connection from the right to the left hand normally serves to calibrate the left hand with the right-hand task-work space. However, when the left- and right-hand spatial maps are in misalignment, the connection mediates realignment of the left-hand spatial map to agree with the right-hand spatial map hand spatial map toward agreement with the right-hand spatial map (Redding \& Wallace, 2002). ${ }^{3}$ That is, intermanual transfer occurs only from the dominant right hand to the nondominant left hand, and only in postexposure testing, when the right-hand task-work space has stabilized sufficiently to specify a left-hand position.

For right-handers, the right-hand spatial map is "dominant," and any spatial misalignment between the two hands is ultimately resolved by realignment of the left-hand spatial map with the right-hand spatial map. If resolution of misalignment in favor of the dominant hand is a function of handedness, then a similar pattern of intermanual transfer could be expected for left-handers, but now with misalignment resolution favoring of the dominant left hand. Our expectation for Experiment 1 was, therefore, that intermanual transfer of the proprioceptive aftereffect would only occur from the dominant left hand to the nondominant right hand, and only when the dominant left hand was tested first after exposure. When the dominant left hand was exposed, the transfer should be positive (i.e., an aftereffect in the nondominant right hand), but when the nondominant right hand was exposed, the transfer should be negative (i.e., a reduction of the aftereffect in the nondominant right hand).

\section{Method}

All 24 subjects were left-handed (see the General Method), and four groups were exposed to rightward prismatic displacement of their vision while pointing with the dominant left hand or the nondominant right hand, with a counterbalanced order of testing the unexposed and exposed hands for proprioceptive shift and total shift. That is, for half of the subjects, the tests were administered in the order proprioceptive shift for the unexposed hand, total shift for the unexposed hand, proprioceptive shift for the exposed hand, total shift for the exposed hand, and finally, visual shift. For the other half of the subjects, the first two pairs of tests were interchanged: proprioceptive shift for the exposed hand, total shift for the exposed hand, proprioceptive shift for the unexposed hand, total shift for the unexposed hand, and finally, visual shift. Subjects pointed

\footnotetext{
$\overline{{ }^{3} \text { For example, }}$ if the right-hand spatial map has been shifted 2 deg rightward, a 2-deg discrepancy exists between straight-ahead in the right-hand and left-hand spatial maps. The 2-deg discrepancy is detected because a spatial map common to all sensory-motor systems mediates communication between the hands (Redding \& Wallace, 1997a). In this common coordinate system, straight-ahead for the right hand is registered as 2 deg to the right, while straight-ahead for the left hand is registered as centered. The spatial discordance is resolved by shifting straight-ahead in the left-hand spatial map 2 deg to the right in the common coordinate system, because the right hand controls calibration of the left hand.
} 
30 times to the rightward-displaced, but objectively straight-ahead, target during the exposure period. This large number of trials was expected to produce overcompensation beyond target achievement. Visual feedback was available early for the distal half of each pointing movement and was expected to produce primarily a proprioceptive aftereffect.

\section{Results and discussion}

Direct effects of the prismatic distortion on performance during exposure are not directly commensurable with aftereffects of prism exposure (but see Redding \& Wallace, 1993). For this reason, the two kinds of measures were analyzed separately, before considering their joint implications. The data are reported in degrees left $(-)$ or right $(+)$ of objective straight-ahead. However, the data may be converted to centimeters at the 74-cm distance to the back of the apparatus by a constant multiplier of 1.31 . For an average reach of $50 \mathrm{~cm}$, the conversion factor is 0.87 .

The main results, presented in detail in the following subsections, were as follows: During prism exposure, terminal error rapidly decreased over exposure trials in largely the same manner for all groups, showing target achievement around halfway through the exposure period, but then showing the expected overcompensation in the second half. Proprioceptive shift was larger than visual shift, as expected from the early visual feedback during exposure, and additivity was present. Also as expected, intermanual transfer of the proprioceptive aftereffect appeared only when the exposed hand was tested first, but contrary to expectation, transfer did not depend on hand dominance.

Exposure performance Performance during exposure was expressed as terminal pointing error, the difference in degrees between terminal finger position and the objective straight-ahead target. An Exposed Hand x Exposure Trial analysis of variance was performed on these terminal error data. The only significant source of variance was the main effect of exposure trial, $F(29,638)=99.00, p<.001$. The sources of variance involving the Exposed Hand factor were not statistically reliable $(p>.332)$. Importantly, exposure adaptation was not affected by any of the grouping variables.

These data are displayed in Fig. 3. As can be seen, terminal error in the rightward direction averaged $5.3 \mathrm{deg} \pm 0.2$ SEM for the first trial, then steadily decreased until the target was achieved by about Trial 13, $0.2 \operatorname{deg} \pm 0.2 S E M$, but then continued in the now antiadaptive leftward direction, showing increasing overshoot of the target for the remaining exposure trials, especially after Trial 20.

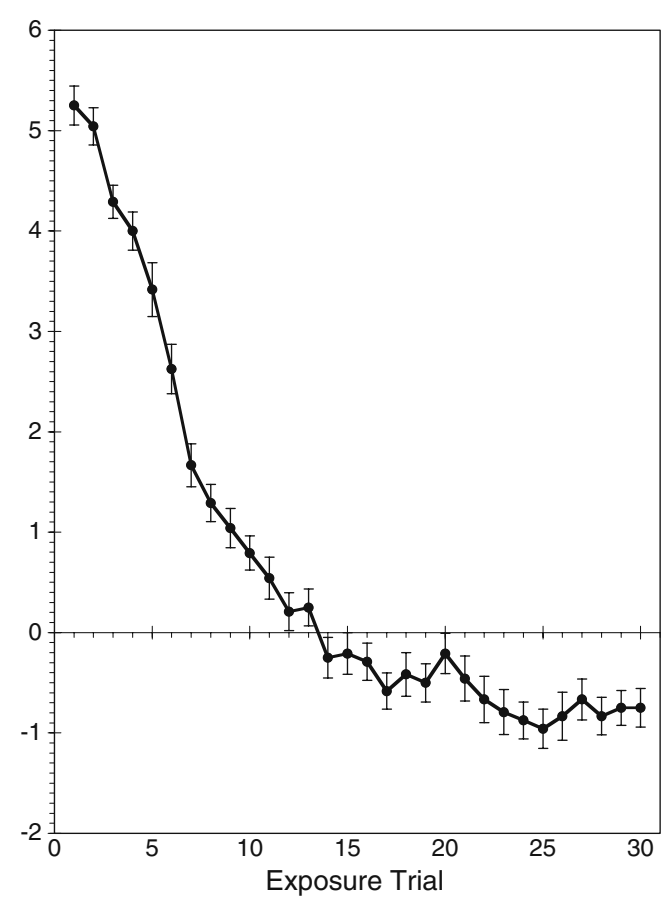

Fig. 3 Experiment 1: Terminal error in exposure target pointing is shown as a function exposure trial. The error bars denote standard errors of the means

Therefore, left-handers showed the same kind of exposure adaptation that right-handers do under the same conditions: rapid target achievement, but developing overcompensation when exposure is continued beyond target achievement, especially when visual feedback is available early in the exposure target pointing (Redding \& Wallace, 1993, 1997b, 2001, 2004, 2006, 2009). This overcompensation has been attributed to the persistence of error-corrective strategies in the face of developing realignment.

Note also that the first-trial "adaptation," $5.3 \mathrm{deg} \pm 0.2$ $S E M$, reflected on the first exposure trial (averaging 54\% of the 11.4-deg prismatic displacement) is also typical of right-handers and can be attributed to ordinary motor undershoot and visual capture that reduces the effective prismatic displacement during exposure (Redding \& Wallace, 2003, 2004).

Component aftereffects Analysis of visual shift and proprioceptive shift aftereffects confirmed that early exposure feedback produced substantial proprioceptive shift in the exposed hand and, therefore, provided the opportunity for intermanual transfer. The only statistically reliable source of variance in this analysis was the main effect of component, $F(1,20)=54.59, p<.001$. No other statistically reliable effects occurred $(p>.455)$.

Proprioceptive shift $(2.4 \mathrm{deg} \pm 0.1$ SEM $)$ was larger than visual shift $(1.1 \mathrm{deg} \pm 0.1 S E M)$. These realignment aftereffect results replicate many previous studies of right- 
handers (e.g., Redding \& Wallace, 1993, 2000; Uhlarik \& Canon, 1971); aftereffects of prismatic displacement are localized in the proprioceptive hand-head system when visual feedback is available early in the exposure pointing movements. Importantly, adaptation was not affected by the manipulation of order of testing. We now turn to transfer measures, which were largely affected by order of testing.

Intermanual transfer Preliminary analysis of the data for the proprioceptive aftereffect test of pointing straight ahead of the nose with the exposed hand revealed no statistically reliable sources of variance $(p>.560)$. Further analysis was, therefore, restricted to the transfer aftereffect test of pointing straight ahead of the nose with the unexposed hand. Any differences found for this measure would reflect differences in intermanual transfer of a statistically constant amount of proprioceptive shift in the exposed hand.

Analysis of variance of the transfer measure revealed a main effect of post exposure test order, $F(1,20)=15.84$, $p=.001$, and an interaction of postexposure test order with exposed hand, $F(1,20)=5.06, p=.036$, but importantly, no main effect for exposed hand, $F(1,20)=0.03, p=.864$. Data for the interaction are displayed in Fig. 4.

On average, transfer was greater when the exposed hand was tested first ( $0.8 \mathrm{deg} \pm 0.14$ SEM) as compared to when the exposed hand was tested second $(0.1 \mathrm{deg} \pm 0.15$ SEM $)$, and this difference was larger when the right hand was exposed (1.0 deg \pm 0.21 SEM vs. $-0.2 \mathrm{deg} \pm 0.17$ SEM)

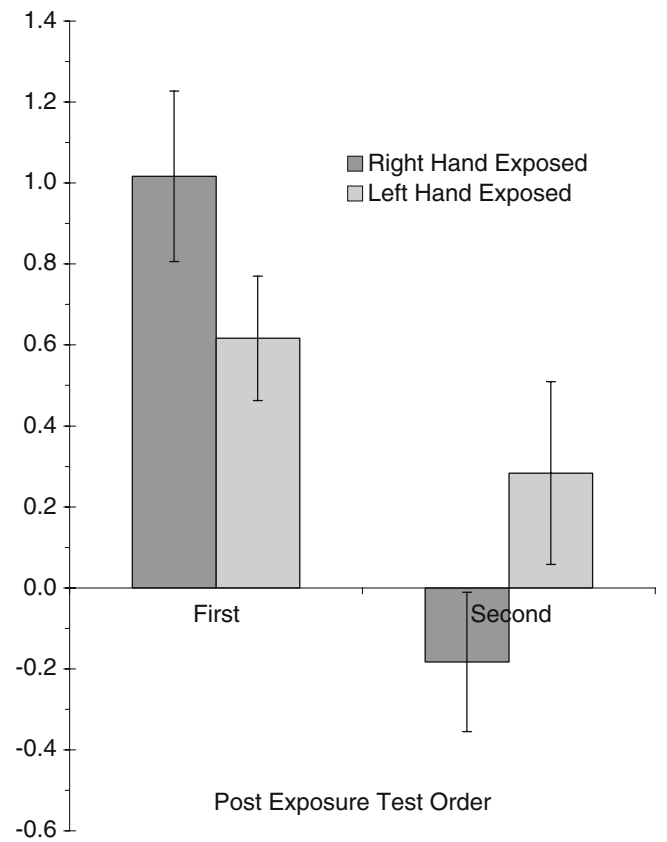

Fig. 4 Experiment 1: Proprioceptive aftereffect for the unexposed limb as a function of which limb was exposed and whether the exposed limb was tested first or second after exposure. The error bars denote standard errors of the means than when the left hand was exposed $(0.6 \mathrm{deg} \pm 0.15$ SEM vs. $0.3 \mathrm{deg} \pm 0.22 S E M$ ). Nevertheless, transfer was statistically reliable when the exposed right hand was tested first, $t(5)=4.86$, two-tailed $p<.005$, and when the exposed left hand was tested first, $t(5)=4.13$, two-tailed $p<.005$. Measures of transfer were not reliably different from zero when the unexposed left hand was tested first, $t(5)=1.06$, two-tailed $p>.500$, or when the unexposed right hand was tested first, $t(5)=1.22$, two-tailed $p>.500$. In summary, transfer occurred when the exposed hand was tested first, but not when the unexposed hand was tested first.

These results suggest that for left-handers, as for righthanders (Redding \& Wallace, 2008), motor control is, at least for purposes of alignment, independent for the two limbs, because intermanual transfer occurred only during postexposure testing, not during exposure. Also, for both left-handers and right-handers, postexposure transfer of adaptation was mediated by a calibration connection between hands. Righthanders have such a connection between the dominant right hand and the nondominant left hand (Redding \& Wallace, 2008). Left-handers also have such a connection between the nondominant right hand and the dominant left hand, but additionally have a connection between the dominant left hand and the nondominant right hand.

For the present purposes, a primary difference between right-handers and left-handers is this additional connection for left-handers. It appears that for left-handers the taskwork space is normally calibrated jointly for the two hands and that resolution of misalignments between the spatial maps for the two hands is a joint function of the two spatial maps; the realignment process ultimately settles on an alignment that reflects part of the misalignment of each spatial map.

For right-handers, the right-hand spatial map determines intermanual alignment, but for left-handers, the two spatial maps are mutually adjusted to arrive at a state of intermanual alignment. For right-handers, the left hand is disadvantaged until realignment is complete, but for lefthanders, both hands are disadvantaged until realignment is complete.

Additivity One of the most firmly established facts of prism adaptation is the additivity of the component aftereffects (e.g., Hay \& Pick, 1966; Hay, 1970; Hay \& Brouchon, 1972; Mikaelian, 1970, 1972, 1974; Redding, 1978; Redding \& Wallace, 1976, 1978, 1990, 1993; Templeton et al., 1974; Wallace \& Garrett, 1975; Wallace \& Redding, 1979; Wilkinson, 1971). That is, visual and proprioceptive aftereffects usually sum to the total aftereffect in the eye-hand coordination loop exercised during exposure. Deviation from additivity is usually traceable to carryover of motor control strategies deployed during exposure (Redding \& Wallace, 1978; Welch et al., 1974). 
Therefore, additivity has become a converging check on the assumption that these aftereffects measure realignment, independent of contributions from strategic control processes such as memory of movements practiced during prism exposure (i.e., motor learning) that might carry over to the postexposure tests (Redding, 1978; Redding \& Wallace, 1988a, 1988b, 1993).

Analysis of the data for the total shift and the sum of the visual shift and proprioceptive shift aftereffect tests found no reliable sources of variance $(p>.478)$. The values for total shift (3.5 deg \pm 0.16 SEM) and the sum of the components (3.5 deg \pm 0.15 SEM) were identical and did not reliably differ as a function of the other variables. Consequently, there is little reason to believe that the validity of the aftereffect measures was compromised in the present experiment.

A similar analysis was performed with the data for the aftereffect measures for the unexposed hand and the visual shift. With these transfer data, the sum of the visual and proprioceptive shifts $(1.5 \mathrm{deg} \pm 0.2$ SEM $)$ was greater than the total shift $(0.8 \mathrm{deg} \pm 0.2 \operatorname{SEM}), F(1,20)=12.60, p=.002$, and this overadditivity did not vary as a function of postexposure test order or exposed hand, $p>.160$. Something more was measured by the sum of components than by target pointing with the unexposed hand. It seems likely that visual realignment did not completely transfer to the unexposed hand, because visual shift was statistically constant across conditions, $p>.316$, while we know from the preceding analysis of intermanual transfer that the measure of proprioceptive transfer did depend on postexposure test order and exposed hand. The second experiment addressed the question of visual shift transfer to target pointing with the unexposed hand.

\section{Experiment 2: Visual transfer}

The incomplete transfer of the visual shift aftereffect found in the first experiment when the total shift aftereffect was measured in the unexposed hand replicated previous findings with right-handers, especially under exposure conditions that promoted visual aftereffects: delayed visual feedback and low response rates (Choe \& Welch, 1974; Cohen, 1967, 1973; Redding \& Wallace, 1988b; Taub \& Goldberg, 1973; Templeton et al., 1974; Wilkinson, 1971). ${ }^{4}$ When visual adaptation was also measured directly to

\footnotetext{
$\overline{{ }^{4} \text { Transfer also }}$ occurs with unconstrained head movement during exposure (Hamilton, 1964), a condition that produces a change in the felt head position (Redding \& Wallace, 1988b, 2003, 2004), which transfers with both the total shift and proprioceptive shift tests (Wallace, 1978)
}

provide a comparison, transfer of visual aftereffects was asymmetric: that is, larger from the nondominant left hand to the dominant right hand (Redding \& Wallace, 1988b).

Redding and Wallace (2009) recently replicated this asymmetry in intermanual transfer of visual aftereffects for right-handers. The aftereffect transferred only to the dominant right hand; that is, visual realignment that occurred with exposure of the left hand also appeared for the unexposed right hand, but realignment with exposure of the right hand did not transfer to the unexposed left hand. Intermanual transfer of the visual aftereffect was asymmetric, occurring only from nondominant to dominant hands.

These results suggest a directional connection from the map of visual space for the nondominant left hand to the map of visual space for the dominant right hand. Realignment of visual space for the left hand transfers to the map of visual space for the right hand, but transfer in the opposite direction does not occur. Such a directional connection is consistent with evidence that the left hand has access to a complete map of visual space, while the right hand directly accesses only right space (e.g., Butler et al., 2004; see also Corbetta, Miezin, Shulman, \& Petersen, 1993; Farnè et al., 2003; see Redding \& Wallace, 2009, for a more extensive review of this research). Right-hand reaches into left space would, therefore, seem to require spatial information from the spatial map of the left hand via a directional connection.

Importantly, Redding and Wallace (2009) also found an asymmetry in the visual aftereffect itself for right-handers that depended on the exposed hand and the direction of prismatic displacement. The aftereffect was smaller in right than in left visual space, but only when the dominant right hand was exposed to leftward displacement. That is, when exposure emphasized right-hand action in left space, realignment was diminished in right space, where action was less frequent. Such diminution did not appear for rightward displacement of the right hand or for the left hand exposed to either direction of displacement.

These results suggest that the representations are separable for left and right visual space, but only for the dominant right hand. Right-hand action in left space accesses only the spatial map for left space. Therefore, the additional spatial map of right space accessed with right-hand action in right space must be specific to the right hand.

The controller for the dominant right hand seems to be specialized for action in right space, with direct access only to a spatial map of that space. Right-hand action in left space accesses the map of left space available from the nondominant left-hand controller, and realignment occurs only for this separate spatial map. The same directional connection responsible for the map of left space employed by the right-hand controller for action in left space also produces asymmetric intermanual transfer of visual realign- 
ment from the left hand to the map of visual space for the right hand.

If these asymmetries in the visual aftereffect are a consequence of dominant-hand specialization, then opposite asymmetries could be expected for left-handers. The second experiment tested the hypotheses that left-handers would show smaller visual aftereffects in left space when the dominant left hand was exposed to rightward displacement and that intermanual transfer of the visual aftereffect would appear only in the dominant left hand.

\section{Method}

All 48 subjects were left-handed (see the General Method), and eight different groups had the dominant left hand or nondominant right hand exposed to leftward or rightward prismatic displacement of vision with counterbalanced orders of testing visual straight-ahead starting in the left and right hemifields: That is, visual shift was tested first in the left-space block of trials or first in the right-space block of trials. The other four tests were administered in the following order: total shift for the exposed hand and then for the unexposed hand, followed by proprioceptive shift for the exposed hand and then for the unexposed hand. Visual feedback during prism exposure was delayed on each of the 20 exposure trials until the terminus of slow, sound-paced target-pointing movement, a condition that was necessary for substantial visual realignment (Redding \& Wallace, 1992, 1993, 1994, 2000; Uhlarik \& Canon, 1971).

\section{Results and discussion}

The main results, presented in detail in the following subsections, were as follows: Decrease in terminal error during exposure was slower with rightward displacement and with the left hand exposed, and overcompensation for the displacement appeared after target achievement. As expected, aftereffects following terminal exposure were almost entirely restricted to the eye-head visual sensorymotor system: That is, visual shift was large and proprioceptive shift near zero, neither variable was affected by the grouping variables, and additivity occurred. Also as expected, visual aftereffects were present for the exposed hands and in transfer for the unexposed dominant left hand, but were largely absent in transfer for the unexposed nondominant right hand. However, contrary to expectation, following leftward displacement of the dominant left hand, visual shift was larger in left space than in right space.

Data analysis An Exposure Trial $\times$ Displacement Direction $x$ Exposed Hand analysis of variance was performed on the terminal error data from prism exposure, expressed in degrees and signed positive in the direction of displacement. Test $\mathrm{x}$ Displacement Direction x Exposed Hand analyses of variance were performed on the aftereffects data, expressed in degrees and signed positive in the direction of adaptive change. The Test factor was different for analyses in the following subsections. Order of the directional tests of visual straight-ahead was an unanalyzed control variable in analyses of both the exposure and aftereffect data.

Exposure performance Exposure performance was expressed as terminal pointing error, the difference in degrees between terminal finger position and the objectively straightahead target. The statistically reliable sources of variance in these data were displacement direction, $F(1,44)=6.55$, $p=.014$, exposed hand, $F(1,44)=17.19, p<.001$, exposure trial, $F(19,836)=244.91, p<.001$, Exposure Trial $\mathrm{x}$ Displacement Direction, $F(19,836)=6.18, p<.001$, and Exposure Trial $\mathrm{x}$ Exposed Hand, $F(19,836)=5.73, p<.001$. No other sources of variance were reliable $(p>.288)$.

Terminal error as a function of exposure trial and displacement direction is illustrated in Fig. 5. As can be seen, the initial errors were $5.0 \mathrm{deg} \pm 0.21$ SEM and $5.1 \mathrm{deg} \pm 0.23$ SEM for leftward and rightward displacement, respectively, and error then declined until the target was achieved by Trial 8 for leftward displacement

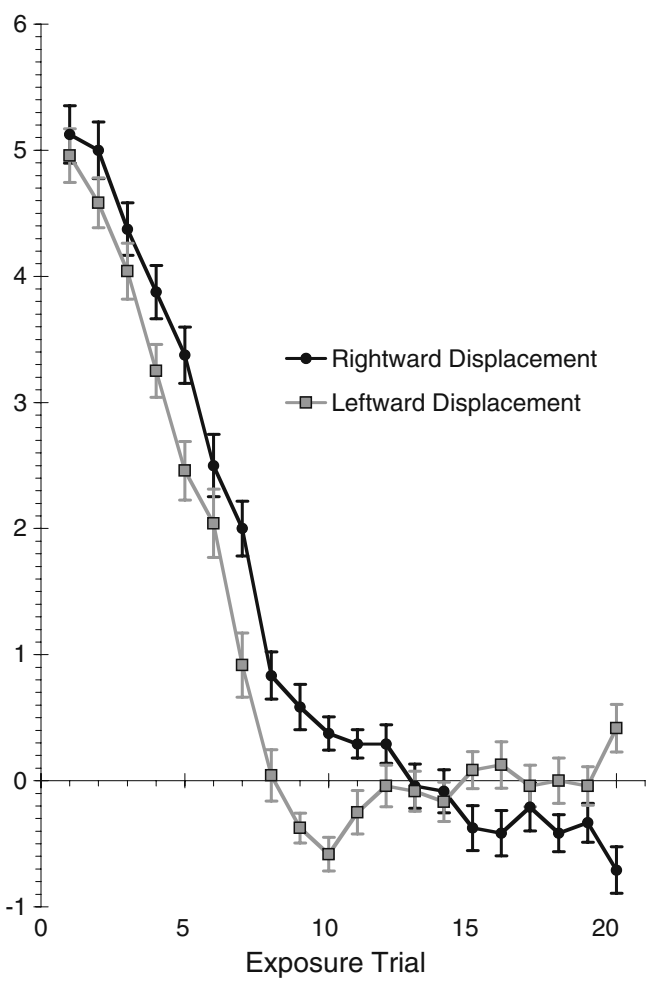

Fig. 5 Experiment 2: Terminal error in exposure target pointing is shown for each of the 20 exposure trials as a function of direction of prismatic displacement. The error bars denote standard errors of the means 
and by Trial 12 for rightward displacement (95\% confidence limits). As in the first experiment, the first-trial "adaptation" (46\% of the 11.4-deg prismatic displacement) can be attributed to ordinary motor undershoot and visual capture that reduces the effective prismatic displacement during exposure (Redding \& Wallace, 2003, 2004). Terminal error was smaller and target achievement faster for leftward than for rightward displacement. On subsequent trials, with leftward displacement, terminal error was reliably different from zero $(95 \%$ confidence limits) only on Trials 9, 10, and 20, but with rightward displacement, terminal error was reliably negative (95\% confidence limits) on Trials 15, 16, 18, 19, and 20. The larger terminal error and slower target achievement with rightward displacement was accompanied by overcompensation for the displacement.

In the similar study of right-handers, Redding and Wallace (2009) did not find statistically reliable modulation of decreasing terminal error for the two directions of displacement. However, reexamination of the data from that experiment showed a pattern similar to the present results, although not as pronounced. Thus, there is no reason to believe that left-handers respond differently from right-handers with respect to displacement direction.

Terminal error as a function of exposure trial and exposed hand is illustrated in Fig. 6. As can be seen, the initial terminal errors were $5.21 \mathrm{deg} \pm 0.23$ SEM for the exposed left hand and $4.9 \mathrm{deg} \pm 0.21 S E M$ for the exposed

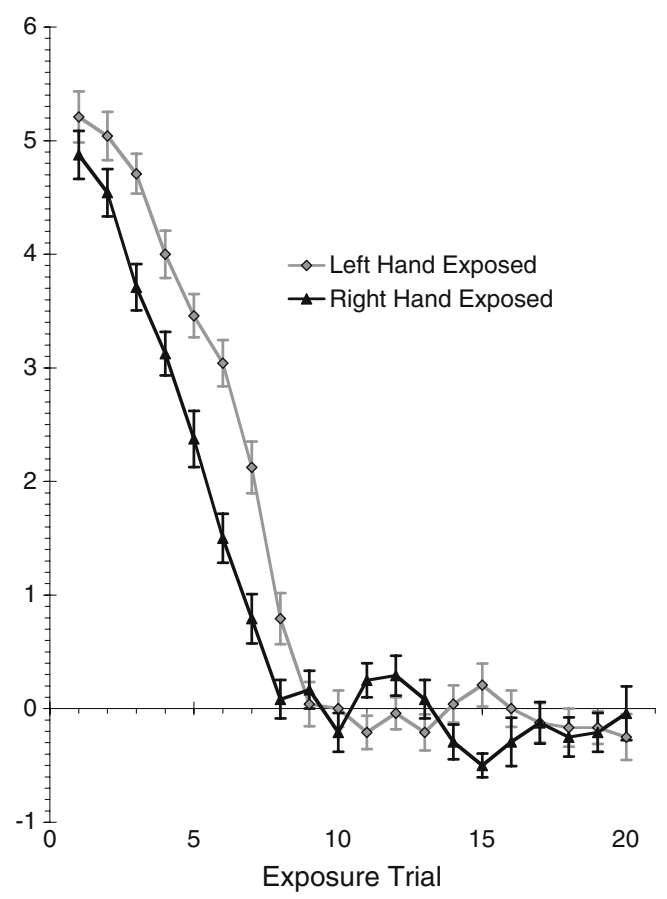

Fig. 6 Experiment 2: Terminal error in exposure target pointing is shown for each of the 20 exposure trials as a function of exposed hand. The error bars denote standard errors of the means right hand. Error then declined until the target was achieved on Trial 9 for the left hand and Trial 8 for the right hand (95\% confidence limits). Target achievement was maintained for all subsequent trials, with the exception of Trial 15 for the right hand, which was reliably negative $(95 \%$ confidence limits). Thus, terminal error was greater and target achievement was slower for the left hand than for the right hand: The smaller terminal error and faster target achievement for the right hand were also accompanied by a tendency toward overcompensation for the displacement.

Reexamination of the data from the comparison study of right-handers (Redding \& Wallace, 2009) showed a pattern of results similar to those of the present study, although it was not statistically reliable. For both studies, terminal error was greater and target achievement slower to occur for the nondominant hand, and the smaller terminal error and faster target achievement for the dominant hand were accompanied by a tendency toward overcompensation. Regardless of handedness, error-corrective strategies were more quickly deployed with the dominant hand, but deployment was also more persistent in the face of developing realignment. Thus, the dominant hand shows an advantage in error correction, but this advantage incurs a liability by its continued application when the strategies become nonadaptive.

Component aftereffects The analysis of visual shift and exposed-hand proprioceptive shift aftereffects confirmed that delayed exposure feedback produced substantial visual shift and, therefore, provided the opportunity for assessing intermanual transfer. The only statistically reliable source of variance in this analysis was the main effect of test, $F(1,44)=830.71, p<.001)$. No other statistically reliable effects occurred $(p>.179)$. Visual shift $(3.00 \mathrm{deg} \pm 0.08$ $S E M)$ was larger than proprioceptive shift $(-0.03 \mathrm{deg} \pm 0.05$ $S E M$ ), and neither measure was affected by any of the between-subjects manipulations.

These component aftereffect data replicate many previous studies of right-handers (e.g., Redding \& Wallace, 1993, 2000; Uhlarik \& Canon, 1971). The aftereffects of prismatic displacement are largely localized in the visual eye-head system when visual feedback is delayed until terminus of the exposure pointing movements.

Additivity The additivity of the aftereffects for the exposedhand data was assessed by comparison of the sum of visual and proprioceptive shift with total shift. There were no significant sources of variance in this analysis. Especially notable were the near-identical values for total shift (3.1 deg \pm 0.09 SEM) and the sum of the component shifts $(3.0 \mathrm{deg} \pm 0.10$ SEM), $F(1,44)=1.85, p=.181$. Additivity appears to have been present for the exposed hand in all conditions, indicating that visual shift transferred completely to the exposed hand. 
The additivity of the components for the exposed hand replicates a predominant outcome for prism adaptation studies in right-handers (Hay, 1970; Hay \& Brouchon, 1972; Redding \& Wallace, 1976, 1988a, 1990; Templeton et al., 1974; Uhlarik \& Canon, 1971; Wallace \& Garrett, 1975; Wallace \& Redding, 1979; Wilkinson, 1971). These results establish that the present conditions substantially replicated those of previous studies of prism adaptation; transfer of error-corrective strategies deployed during exposure did not compromise the aftereffect measures.

Transfer A similar additivity-of-aftereffects analysis was performed on the data for the unexposed hand, except that the levels of the test variable were given by target pointing with the unexposed hand, and the components were visual shift and proprioceptive shift in the unexposed hand. The statistically reliable sources of variance were unexposed hand, $F(1,44)=90.18, p<.001$, test, $F(1,44)=79.49, p<.001$, Test $\mathrm{x}$ Displacement Direction, $F(1,44)=8.67, p=.005$, and Test $\mathrm{x}$ Unexposed Hand, $F(1,44)=128.16, p<.001$.

Data for the transfer measures as a function of unexposed hand are displayed on the left in Fig. 7. As can be seen, the total shift aftereffect for target pointing with the unexposed hand closely matched the sum of components only for the unexposed left hand. The over-

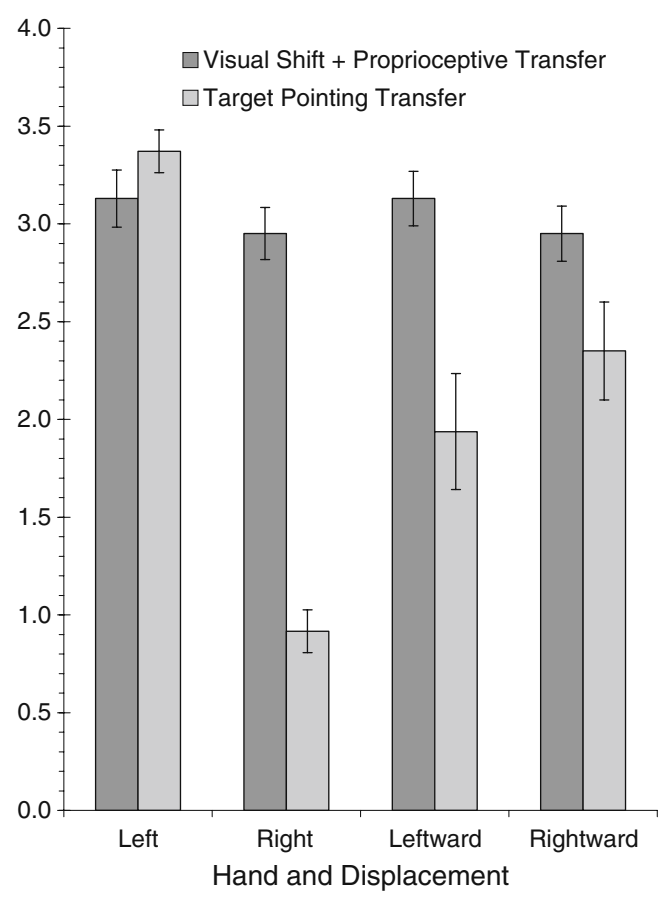

Fig. 7 Experiment 2: Transfer of visual realignment to the unexposed hand. The sum of the visual shift aftereffect and the proprioceptive transfer aftereffect for the unexposed hand is compared to the total shift aftereffect of target pointing with the unexposed hand, first as a function of which hand was unexposed (left two graphs) and second as a function of displacement direction (right two graphs). The error bars denote standard errors of the means additivity for the unexposed right hand indicates incomplete transfer of visual adaptation. As also shown in Fig. 7, this overadditivity was present for both directions of displacement, but somewhat larger for leftward than for rightward displacement.

For these left-handed subjects, visual adaptation acquired during exposure of the nondominant right hand transferred to the unexposed dominant left hand, but visual adaptation acquired with the exposure of the dominant left hand did not transfer to the unexposed nondominant right hand. In comparison, Redding and Wallace (2009) found for righthanders that visual adaptation acquired during exposure of the nondominant left hand transferred to the unexposed dominant right hand, but visual adaptation acquired with exposure of the dominant right hand did not transfer to the unexposed nondominant left hand. In summary, regardless of handedness, visual adaptation acquired during exposure of the nondominant hand transfers to the unexposed dominant hand, but visual adaptation acquired with exposure of the dominant hand does not transfer to the unexposed nondominant hand.

\section{Directional visual shift}

Directional aftereffects for visual shift were assessed by a comparison of the aftereffects in left and right space. This analysis included the following statistically significant sources of variance: Test $\times$ Displacement Direction, $F(1,44)=9.39, p=.004$, and Test $\times$ Exposed Hand, $F(1,44)=10.95, p=.002$. Importantly, the threeway interaction of Displacement Direction $\times$ Exposed Hand $\times$ Direction of Test was not statistically reliable, $F(1,44)=1.82, p=.184$. No other effects were statistically reliable, $p>.107$.

When the dominant left hand was exposed, the visual aftereffect was larger in left space (3.0 deg $\pm 0.1 S E M$ vs. $2.8 \mathrm{deg} \pm 0.1$ SEM), but when the nondominant right had was exposed, the visual aftereffect was larger in right space (3.2 deg $\pm 0.1 S E M$ vs. $3.0 \mathrm{deg} \pm 0.1$ SEM). With leftward displacement, the visual aftereffect was larger in left space (3.2 deg $\pm 0.1 S E M$ vs. $3.0 \mathrm{deg} \pm 0.1 S E M$ ), but with rightward displacement, the visual aftereffect was larger in right space (3.0 deg $\pm 0.1 S E M$ vs. $2.8 \mathrm{deg} \pm 0.1 S E M$ ).

These results contrast with those of Redding and Wallace (2009) for right-handers, in which the asymmetry in visual straight-ahead was a joint function of exposed hand and displacement direction. This difference in outcomes justified a comparison of the left- and right-space tests for the four groups, which indicated that with leftward displacement of the left hand, the adaptive shift in visual straightahead was less in right space (2.6 deg \pm 0.2 SEM) than in left space (3.2 deg \pm 0.2 SEM), $t(11)=5.38, p<.001$, while 
all of the other groups, on average, tended to show the opposite asymmetry, $t(35)=2.15, p=.037(3.0 \mathrm{deg} \pm 0.1$ $S E M$ vs. $3.1 \mathrm{deg} \pm 0.1 S E M$ ).

For left-handers, the asymmetry in visual realignment between left and right space appeared when the dominant left hand was exposed to leftward displacement. In comparison, for right-handers, Redding and Wallace (2009) found that asymmetry in visual realignment between left and right space appeared when the dominant right hand was exposed to leftward displacement. Regardless of handedness, then, asymmetry in visual realignment between left and right space appears when the dominant hand is exposed to leftward displacement.

\section{Conclusions}

Left-handers adapt to prismatic displacement in much the same way as right-handers. There were no apparent differences in performance during exposure (direct effects), and regardless of handedness, either or both visual and proprioceptive aftereffects occurred, depending on the visual feedback delay during exposure. These component aftereffects are additive and equal the total aftereffect for the eye-hand coordination loop. The two hands are controlled independently for both left- and right-handers. However, notable and somewhat paradoxical differences in the intermanual transfer of adaptation do appear between left- and right-handers.

For both left-handers and right-handers, visual adaptation acquired during exposure of the nondominant hand transfers to the unexposed dominant hand, but visual adaptation acquired with exposure of the dominant hand does not transfer to the unexposed nondominant hand. Moreover, regardless of handedness, an asymmetry in visual adaptation between left and right space appears when the dominant hand is exposed to leftward displacement (Exp. 2 above; Redding \& Wallace, 2009).

These last two conclusions, however, present an interesting paradox. Left and right space appear to be separable for the dominant hand, and the nondominant hand appears to be directionally connected to the dominant hand, but the dominant hand is physiologically and neurologically different for left- and right-handers!

One possible resolution of this puzzle, illustrated in Fig. 8, might be that the organization of the visual system is the same for both left- and right-handers, but that connections to the independent controllers for the left and right hands are reversed for left-handers and right-handers. The connections of the visual system to the right-hand controller that exist in right-handers are made to the lefthand controller in left-handers. Therefore, differential visual adaptation for the dominant and nondominant hands is the same, regardless of handedness.

\section{Right-Handers}

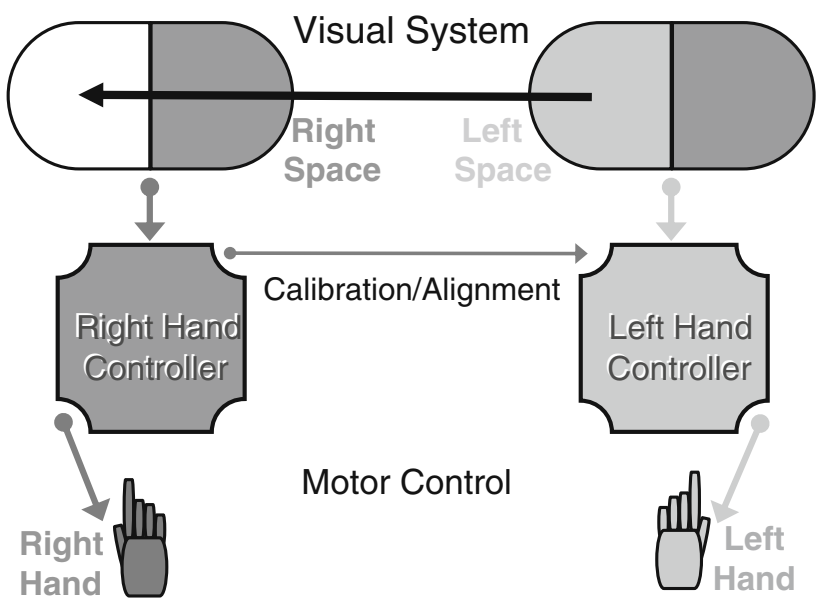

\section{Left-Handers}

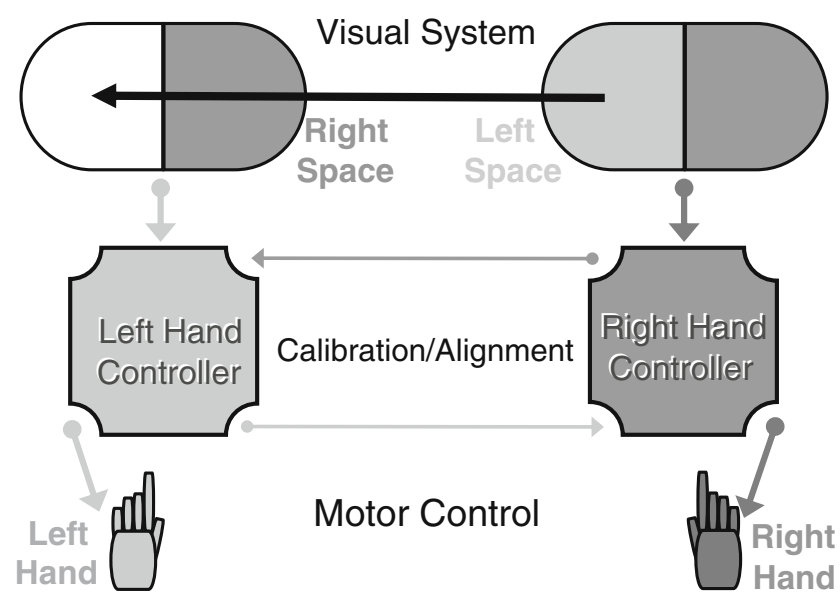

Fig. 8 The differential motor organization proposed for right-handers and left-handers. Regardless of handedness, the organization of the visual system is the same, as shown in the top portion of each illustration, with a complete map of visual space for the nondominant hand, a representation only of right space for the dominant hand, and a directional connection from nondominant to dominant controllers in order to enable dominant-hand reaches into left space; this directional connection mediates the asymmetric transfer and magnitudes of visual adaptation. The motor system is shown differently organized for handedness, as shown in the bottom portion of each illustration: For right-handers, the connection that normally serves to calibrate the left hand for the right-hand task-work space also mediates transfer of realignment when the two spatial maps are in misalignment. For lefthanders, the two hands are jointly calibrated for the current task-work space, and when spatial misalignment occurs, realignment occurs for both spatial maps

Such a difference in motor control of the two limbs might also be extended to explain the paradoxical difference found for intermanual transfer of proprioceptive adaptation between left-handers and right-handers. Regardless of handedness, proprioceptive adaptation of the dominant hand transfers to the nondominant hand during 
postexposure testing. However, postexposure transfer also occurs from the nondominant to the dominant hand for lefthanders, but not for right-handers (Exp. 1 above; Redding $\&$ Wallace, 2008). Again these results are puzzling, because the dominant hand is physiologically and neurologically different for left- and right-handers. As illustrated in the bottom portion of Fig. 8, for left-handers it appears that both hands jointly set the present task-work space and that the bidirectional calibration connections between hands mediate intermanual transfer proprioceptive realignment in both directions.

The suggestion that handedness is primarily a consequence of differential motor control provides a parsimonious explanation of the data from prism adaptation. We make no claim that this is a complete account of handedness. However, the results from prism adaptation should be taken into account in the formulation of any theory of handedness.

\section{References}

American Psychological Association. (1992). Ethical principles of psychologists and code of conduct. The American Psychologist, 47, 1597-1611.

Aschersleben, G., Drewing, K., \& Stenneken, P. (2002). Temporal coordination of simple movements. Cognitive Processing, 1-2, $37-60$.

Beckett, P. A. (1980). Development of the third component of prism adaptation: Effects of active and passive movement. Journal of Experimental Psychology. Human Perception and Performance, 6, 433-444.

Bedford, F. L. (1989). Constraints on learning new mappings between perceptual dimensions. Journal of Experimental Psychology. Human Perception and Performance, 15, 232-248.

Bedford, F. (1993a). Perceptual and cognitive spatial learning. Journal of Experimental Psychology. Human Perception and Performance, 19, 517-530.

Bedford, F. (1993b). Perceptual learning. In D. Medin (Ed.), The psychology of learning and motivation (Vol. 30, pp. 1-60). Orlando, FL: Academic Press.

Bedford, F. L. (1999). Keeping perception accurate. Trends in Cognitive Sciences, 3, 4-11.

Butler, A. J., Fink, G. R., Dohle, C., Wunderlich, G., Tellmann, L., Seitz, R. J., et al. (2004). Neural mechanisms underlying reaching for remembered targets cued kinesthetically or visually in left or right hemispace. Human Brain Mapping, 21, 165-177.

Canon, L. K. (1970). Intermodality inconsistency of input and directed attention as determinant of the nature of adaptation. Journal of Experimental Psychology, 84, 141-147.

Choe, C. S., \& Welch, R. B. (1974). Variables affecting the intermanual transfer and decay of prism adaptation. Journal of Experimental Psychology, 102, 1076-1084.

Churchland, P. S. (1986). Neurophilosophy: Toward a unified science of the mind-brain. Cambridge, MA: MIT Press.

Cohen, M. M. (1967). Continuous versus terminal visual feedback in prism aftereffects. Perceptual and Motor Skills, 24, 1295-1302.

Cohen, M. M. (1973). Visual feedback, distribution of practice, and intermanual transfer of prism aftereffects. Perceptual and Motor Skills, 37, 599-609.
Corbetta, M., Miezin, F. M., Shulman, G. L., \& Petersen, S. E. (1993). A PET study of visuospatial attention. The Journal of Neuroscience, 13, 1202-1226.

Craske, B. (1967). Adaptation to prisms: Change in internally registered eye-position. British Journal of Experimental Psychology, 58, 329-335.

Crawshaw, M., \& Craske, B. (1974). No retinal component in prism adaptation. Acta Psychologica, 38, 421-423.

Farnè, A., Roy, A. C., Paulignan, Y., Rode, G., Rossetti, Y., Boisson, D., et al. (2003). Visuo-motor control of the ipsilateral hand: Evidence from right brain-damaged patients. Neuropsychologia, 41, 739-757. doi:10.1016/S0028-3932(02)00177-X

Hamilton, C. R. (1964). Intermanual transfer of adaptation to prisms. The American Journal of Psychology, 77, 457-462.

Harris, C. S. (1963). Adaptation to displaced vision: Visual, motor, or proprioceptive change? Science, 140, 812-813.

Harris, C. S. (1965). Perceptual adaptation to inverted, reversed, and displaced vision. Psychological Review, 72, 419-444.

Harris, C. S. (1980). Insight or out of sight? Two examples of perceptual plasticity in the human adult. In C. S. Harris (Ed.), Visual coding and adaptability (pp. 95-150). Hillsdale, NJ: Erlbaum.

Hay, L. (1970). Contribution à l'étude de l'organisation de l'espace postural chez l'Homme: Étude expérimentale de l'adaptation des différents segments du corps à une déviation prismatique du champ visuel [Contribution to the study of the organization of postural space in man: Experimental study of the adaptation of different body segments to a prismatic displacement of the visual field]. Cahiers de Psychologie, 13, $3-24$.

Hay, L., \& Brouchon, M. (1972). Analyse de la réorganisation des coordinations visuo-motrices chez l'homme [Analysis of the reorganization of visual-motor coordination in man]. L'Année Psychologique, 72, 25-38.

Hay, J. C., \& Pick, H. L., Jr. (1966). Visual and proprioceptive adaptation to optical displacement of the visual stimulus. Journal of Experimental Psychology, 71, 150-158.

Held, R., \& Hein, A. (1958). Adaptation to disarranged hand-eye coordination contingent upon reafferent stimulation. Perceptual and Motor Skills, 8, 87-90.

Jeannerod, M. (1988). The neural and behavioral organization of goal-directed movements. Oxford: Oxford University Press, Clarendon Press.

Kelso, J. A. S., Cook, E., Olson, M. E., \& Epstein, W. (1975). Allocation of attention and the locus of adaptation to displaced vision. Journal of Experimental Psychology. Human Perception and Performance, 1, 237-245.

McLaughlin, S. C., \& Webster, R. G. (1967). Changes in straightahead eye position during adaptation to wedge prisms. Perception \& Psychophysics, 2, 37-44.

Mikaelian, H. H. (1970). Adaptation to rearranged eye-foot coordination. Perception \& Psychophysics, 8, 222-224.

Mikaelian, H. H. (1972). Lack of bilateral generalization of adaptation to auditory rearrangement. Perception \& Psychophysics, 11, 222224.

Mikaelian, H. H. (1974). Adaptation to displaced hearing: A nonproprioceptive change. Journal of Experimental Psychology, 103, 326-330.

Newport, R., \& Jackson, S. R. (2006). Posterior parietal cortex and the dissociable components of prism adaptation. Neuropsychologia, 44, 2757-2765.

Oldfield, R. (1971). The assessment and analysis of handedness: The Edinburgh inventory. Neuropsychologia, 9, 97-113.

Prablanc, C., Tzavaras, T., \& Jeannerod, M. (1975). Adaptation of the two arms to opposite prism displacements. The Quarterly Journal of Experimental Psychology, 27, 667-671. 
Redding, G. M. (1978). Additivity in adaptation to optical tilt. Journal of Experimental Psychology. Human Perception and Performance, 4, 178-190.

Redding, G. M., Clark, S. E., \& Wallace, B. (1985). Attention and prism adaptation. Cognitive Psychology, 17, 1-25.

Redding, G. M., Rossetti, Y., \& Wallace, B. (2005). Applications of prism adaptation: A tutorial in theory and method. Neuroscience and Biobehavioral Reviews, 29, 431-444.

Redding, G. M., \& Wallace, B. (1976). Components of displacement adaptation in acquisition and decay as a function of hand and hall exposure. Perception \& Psychophysics, 20, 453-459.

Redding, G. M., \& Wallace, B. (1978). Sources of "overadditivity" in prism adaptation. Perception \& Psychophysics, 24, 58-62.

Redding, G. M., \& Wallace, B. (1988a). Adaptive mechanisms in perceptual-motor coordination: Components of prism adaptation. Journal of Motor Behavior, 20, 242-254.

Redding, G. M., \& Wallace, B. (1988b). Components of prism adaptation in terminal and concurrent exposure: Organization of the eye-hand coordination loop. Perception \& Psychophysics, 44, 59-68.

Redding, G. M., \& Wallace, B. (1990). Effects on prism adaptation of duration and timing of visual feedback during pointing. Journal of Motor Behavior, 22, 209-224.

Redding, G. M., \& Wallace, B. (1992). Effects of pointing rate and availability of visual feedback on visual and proprioceptive components of prism adaptation. Journal of Motor Behavior, 24, 226-237.

Redding, G. M., \& Wallace, B. (1993). Adaptive coordination and alignment of eye and hand. Journal of Motor Behavior, 25, 7588.

Redding, G. M., \& Wallace, B. (1994). Effects of movement duration and visual feedback on visual and proprioceptive components of prism adaptation. Journal of Motor Behavior, 26, 257-266.

Redding, G. M., \& Wallace, B. (1996). Adaptive spatial alignment and strategic perceptual-motor control. Journal of Experimental Psychology. Human Perception and Performance, 22, 379394.

Redding, G. M., \& Wallace, B. (1997a). Adaptive spatial alignment. Mahwah, NJ: Erlbaum.

Redding, G. M., \& Wallace, B. (1997b). Prism adaptation during target pointing from visible and nonvisible starting locations. Journal of Motor Behavior, 29, 119-130.

Redding, G. M., \& Wallace, B. (1998). Phenomenal versus process explanations of prism aftereffects. Journal of Motor Behavior, 30, 44-50.

Redding, G. M., \& Wallace, B. (2000). Prism exposure aftereffects and direct effects for different movement and feedback times. Journal of Motor Behavior, 32, 83-99.

Redding, G. M., \& Wallace, B. (2001). Calibration and alignment are separable: Evidence from prism adaptation. Journal of Motor Behavior, 33, 401-412.

Redding, G. M., \& Wallace, B. (2002). Strategic calibration and spatial alignment: A model from prism adaptation. Journal of Motor Behavior, 34, 126-138.

Redding, G. M., \& Wallace, B. (2003). First trial "adaptation" to prism exposure. Journal of Motor Behavior, 35, 229-245.

Redding, G. M., \& Wallace, B. (2004). First trial "adaptation" to prism exposure: Artifact of visual capture. Journal of Motor Behavior, 36, 291-304.
Redding, G. M., \& Wallace, B. (2006). Generalization of prism adaptation. Journal of Experimental Psychology. Human Perception and Performance, 32, 1006-1022.

Redding, G. M., \& Wallace, B. (2008). Intermanual transfer of prism adaptation. Journal of Motor Behavior, 40, 246-262.

Redding, G. M., \& Wallace, B. (2009). Asymmetric visual prism adaptation and intermanual transfer. Journal of Motor Behavior, $41,83-96$.

Rock, I., \& Harris, C. S. (1967). Vision and touch. Scientific American, 216, 96-104.

Simani, M. C., McGuire, L. M. M., \& Sabes, P. N. (2007). Visual-shift adaptation is composed of separable sensory and task-dependent effects. Journal of Neurophysiology, 98, 2827-2841.

Taub, E., \& Goldberg, I. A. (1973). Prism adaptation: Control of intermanual transfer by distribution of practice. Science, 180, $755-757$.

Templeton, W. B., Howard, I. P., \& Wilkinson, D. A. (1974). Additivity of components of prismatic adaptation. Perception \& Psychophysics, 15, 249-257.

Uhlarik, J. J. (1973). Role of cognitive factors on adaptation to prismatic displacement. Journal of Experimental Psychology, 98, 223-232.

Uhlarik, J. J., \& Canon, L. K. (1971). Influence of concurrent and terminal exposure conditions on the nature of perceptual adaptation. Journal of Experimental Psychology, 9, 233-239.

von Helmholtz, H. E. F. (1962). Treatise on physiological optics (J. P. C. Southall, Ed. and Trans.). New York: Dover. (Original work published 1909)

Wallace, B. (1974). Target and arm observation effects on adaptation to prism displacement. Perception \& Psychophysics, 15, 145-148.

Wallace, B. (1977). Stability of Wilkinson's linear model of prism adaptation over time for various targets. Perception, 6, 145-151.

Wallace, B. (1978). Visuomotor coordination and intermanual transfer for a proprioceptive reaching task. Journal of Motor Behavior, 10, 139-147.

Wallace, B., \& Garrett, J. B. (1975). Perceptual adaptation with selective reductions of felt sensation. Perception, 4, 437-445.

Wallace, B., \& Redding, G. M. (1979). Additivity in prism adaptation as manifested in intermanual and interocular transfer. Perception \& Psychophysics, 25, 133-136.

Weiner, M. J., Hallett, M., \& Funkenstein, H. H. (1983). Adaptation to lateral displacement of vision in patients with lesions of the central nervous system. Neurology, 33, 766-772.

Welch, R. B. (1971). Discriminative conditioning of prism adaptation. Perception \& Psychophysics, 10, 90-92.

Welch, R. B. (1978). Perceptual modification: Adapting to altered sensory environments. New York: Academic Press.

Welch, R. B. (1986). Adaptation of space perception. In K. R. Boff, L. Kaufman, \& J. R. Thomas (Eds.), Handbook of perception and human performance: Vol. 1. Sensory processes and perception (pp. 24.1-24.45). New York: Wiley.

Welch, R. B., Choe, C. S., \& Heinrich, D. R. (1974). Evidence for a three-component model of prism adaptation. Journal of Experimental Psychology, 103, 700-705.

Welch, R. B., \& Rhodes, R. W. (1969). The manipulation of informational feedback and its effects upon prism adaptation. Canadian Journal of Psychology, 23, 415-428.

Wilkinson, D. A. (1971). Visual-motor control loop: A linear system? Journal of Experimental Psychology, 89, 250-257. 